\title{
DISCRETE AND DENSE SUBGROUPS ACTING ON COMPLEX HYPERBOLIC SPACE
}

\author{
WENSHENG CAO
}

(Received 29 October 2007)

\begin{abstract}
In this paper, we study the discreteness criteria for nonelementary subgroups of $\mathrm{U}(1, n ; \mathbb{C})$ acting on complex hyperbolic space. Several discreteness criteria are obtained. As applications, we obtain a classification of nonelementary subgroups of $\mathrm{U}(1, n ; \mathbb{C})$ and show that any dense subgroup of $\mathrm{SU}(1, n ; \mathbb{C})$ contains a dense subgroup generated by at most $n$ elements when $n \geq 2$. We also obtain a necessary and sufficient condition for the normalizer of a discrete and nonelementary subgroup in $\mathrm{SU}(1, n ; \mathbb{C})$ to be discrete.
\end{abstract}

2000 Mathematics subject classification: primary 30F40; secondary 20H10.

Keywords and phrases: discreteness criterion, nonelementary subgroup, dense subgroup, normalizer.

\section{Introduction}

The discreteness of Möbius groups is a fundamental problem which has been extensively studied. In 1976, by using the so-called Jørgensen inequality, Jørgensen [9] established the following well-known result.

THEOREM J. A nonelementary subgroup $G$ of $\mathrm{SL}(2, \mathbb{C})$ is discrete if and only if all its two-generator subgroups are discrete.

Martin [14] generalized Theorem J to the $n$-dimensional Möbius group $M(n)$.

THEOREM M. A nonelementary and finitely generated subgroup $G$ of $M(n)$ is discrete if and only if all its two-generator subgroups are discrete.

See, for example, $[18,19]$ for further generalizations of Theorems $\mathrm{J}$ and $\mathrm{M}$ in $M(n)$.

It follows from a result of Sullivan (see [17, p. 246]) that a nonelementary and nondiscrete subgroup $G$ of $\operatorname{SL}(2, \mathbb{C})$ is either conjugate to a dense subgroup of $\operatorname{SL}(2, \mathbb{R})$, or dense in $\operatorname{SL}(2, \mathbb{C})$. Wang generalized the above result to the case of $M(n)$. Using this generalized result, Wang showed that any dense subgroup of

This research was partly supported by NSF (no: 10671004) of China and the doctoral start-up science foundation of Guangdong Province.

(C) 2008 Australian Mathematical Society 0004-9727/08 \$A2.00+0.00 
$M(n)$ contains a dense subgroup which is generated by at most $n$ elements if $n \geq 2$ and, if $n=1$, by two elements. See [18] for details.

Let $G$ be a discrete and nonelementary subgroup of $M(n)$ and $\mathcal{N}(G)$ the normalizer of $G$ in $M(n)$, that is,

$$
\mathcal{N}(G)=\left\{g \in M(n) \mid g G g^{-1}=G\right\} .
$$

Katok (see [11, p. 36]) and Maskit (see [13, p. 98]) proved that $G$ and $\mathcal{N}(G)$ share discreteness for $n=1$ and $n=2$, respectively. Ratcliffe [16] proved this for a finitely generated nonelementary group $G \in M(n)$ with the property that the hull of its limit set has full dimension. Wang [20] proved the following result.

THEOREM W. Let $G$ be a nonelementary subgroup of $M(n)$. Then $\mathcal{N}(G)$ is discrete if and only if both $G$ and $W(\mathcal{N}(G))$ are discrete.

Here

$$
W(G)=\bigcap_{f \in h(G)} G_{\mathrm{fix}(f)},
$$

where $h(G)$ is the set of all loxodromic elements in $G$ and $G_{\text {fix }(f)}=\{g \in G \mid \operatorname{fix}(f)$ $\subset \operatorname{fix}(g)\}$.

Because of the closed connection between real and complex hyperbolic geometry, the roadmap of analogy frequently points the way toward potentially interesting questions. The complex hyperbolic space is more complicated than the real hyperbolic space. For example, it has variable negative curvature, it is a Kähler manifold with biholomorphic automorphisms and its boundary has a natural contact structure which is locally modelled on Heisenberg geometry (see [1, 12]). It is interesting to investigate analogous results of real hyperbolic space in the setting of complex hyperbolic space.

There has been much research in this area; see, for example, [2, 5, 8, 15]. Our purpose in this paper is to find analogous results mentioned above in the setting of complex hyperbolic space. In order to state our main results, we first recall some notation and facts about complex hyperbolic space.

The complex hyperbolic $n$-space $H_{\mathbb{C}}^{n}$ may be identified with the unit ball in $\mathbb{C}^{n}$ with the Bergman metric. The group of its holomorphic isometries is the group $\mathrm{U}(1, n ; \mathbb{C})$ acting on $H_{\mathbb{C}}^{n}$ and on its boundary $\partial H_{\mathbb{C}}^{n}$. A nontrivial element $g$ of $\mathrm{U}(1, n ; \mathbb{C})$ is said to be elliptic if it has a fixed point in $H_{\mathbb{C}}^{n}$, parabolic if it has exactly one fixed point and this lies on $\partial H_{\mathbb{C}}^{n}$, and loxodromic if it has exactly two fixed points and they lie on $\partial H_{\mathbb{C}}^{n}$. The limit set $L(G)$ of the subgroup $G \subset U(1, n ; \mathbb{C})$ is defined as

$$
L(G)=\overline{G(p)} \cap \partial H_{\mathbb{C}}^{n}, \quad p \in H_{\mathbb{C}}^{n}
$$

A subgroup $G$ of $\mathrm{U}(1, n ; \mathbb{C})$ is called nonelementary if it contains two nonelliptic elements of infinite order with distinct fixed points; otherwise $G$ is called elementary. We refer to $[7,8]$ for more details of these concepts and some of their properties. 
Using the same notation as in [7], for any totally geodesic submanifold $M \subset H_{\mathbb{C}}^{n}$, we denote by $I(M)$ the subgroup of $\mathrm{U}(1, n ; \mathbb{C})$ which leaves $M$ invariant and by $K(M)$ the subgroup of $I(M)$ which leaves $M$ pointwise fixed. Then there exists a Lie subgroup $\mathrm{U}(M) \subset I(M)$ such that

$$
I(M)=K(M) U(M) \quad \text { with } K(M) \cap U(M) \text { finite. }
$$

In particular, if $M=H_{\mathbb{C}}^{m}$ is viewed as an embedding totally geodesic submanifold of $H_{\mathbb{C}}^{n}$, then

$$
K(M)=\omega I_{m+1} \times U(n-m ; \mathbb{C}), \quad U(M)=\mathrm{SU}(1, m ; \mathbb{C}) \times I_{n-m},
$$

where $\omega$ is a complex number with norm $1, I_{k}$ is the $k \times k$ unit matrix, and $\mathrm{SU}(1, m ; \mathbb{C})$ consists of matrices in $\mathrm{U}(1, m ; \mathbb{C})$ with determinant 1 .

The smallest totally geodesic submanifold $M(G)$, invariant under $G$, coincides with the smallest totally geodesic submanifold whose boundary contains $L(G)$ when the cardinality of the limit set $L(G)$ is at least 1 . If $f \in G$ fixes $L(G)$ pointwise, then $f$ fixes $M(G)$ pointwise. Let $\partial M(G)=\overline{M(G)} \cap \partial H_{\mathbb{C}}^{n}$. Then $L(G) \subset \partial M(G)$ and $G \subset I(M(G))$.

From the group theory point of view, we can study the group $G \cap K(M(G))$ and its coset spaces in $G$ to reveal some properties of $G$. It is expedient to introduce the following map $\phi$ to realize its coset spaces. Let $\phi(g)$ be the restriction of $g$ to $M(G)$ and let $\phi(G)$ consist of $\phi(g)$ for all $g \in G$, that is,

$$
\phi(g)=\left.g\right|_{M(G)}, \quad \phi(G)=\left\{\left.g\right|_{M(G)}: g \in G\right\} .
$$

Then $\phi$ is a homomorphism from $G$ onto $\phi(G)$ and the kernel $\operatorname{ker}(\phi)$ of $\phi$ consists of the elements of $G$ whose restriction $\left.g\right|_{M(G)}$ is identity, that is, $\operatorname{ker}(\phi)=G \cap$ $K(M(G))$. Sometimes we denote the $\phi$ by $\phi_{G}$ to emphasize the dependence of $\phi$ on $G$. Since $\phi(G)$ and $G$ have the same orbits in $M(G)$,

$$
L(\phi(G))=L(G)
$$

and $G$ is nonelementary if and only if $\phi(G)$ is nonelementary.

We denote by $\mathrm{U}(1, M(G))$ the restriction of $\mathrm{U}(1, n ; \mathbb{C})$ on $M(G)$ which leaves $M(G)$ invariant. We mention that the elements of $\phi(G) \subset U(1, M(G))$ can be represented by suitable matrices in the general linear group $G L(d+1, \mathbb{C})$, where $d=\operatorname{dim}(M(G))$. For instance, we can view $\mathrm{U}\left(1, H_{\mathbb{C}}^{m}\right)$ as $\mathrm{U}(1, m ; \mathbb{C})$ and $\phi_{G_{1}}(g)$ and $\phi_{G}(g)$ may not be of the same size as matrices for $g \in G_{1} \subset G$. It is obvious that we can always conjugate $\mathrm{U}(1, M(G))$ to $\mathrm{U}(1, d ; \mathbb{C})$ in $\mathrm{U}(1, n ; \mathbb{C})$.

For a nonelementary subgroup $G \subset U(1, n$; $\mathbb{C})$, let

$$
\mathcal{H}(G)=\{f \in G \mid f \text { is loxodromic }\},
$$

and if $G$ contains some parabolic element, then let

$$
\mathcal{P}(G)=\{f \in G \mid f \text { is parabolic }\} .
$$


For $f \in \mathcal{H}(G)$, or $\mathcal{P}(G)$ if $\mathcal{P}(G) \neq \emptyset$, let

$$
G_{f}=\{g \in G \mid g \text { is conjugate to } f \text { and }\langle f, g\rangle \text { is nonelementary }\} .
$$

Our main results concerning discreteness are the following theorems.

THEOREM 1.1. Let $G \subset U(1, n ; \mathbb{C})$ be nonelementary and $\phi(G)$ be as in (2). Then $\phi(G)$ is discrete if and only if for every two-generator, nonelementary $G_{\beta}$ of $\phi(G)$, $\phi\left(G_{\beta}\right)$ is discrete.

TheOREM 1.2. Let $G \subset U(1, n ; \mathbb{C})$ be nonelementary. Then $G$ is discrete if and only if $\operatorname{ker}(\phi)$ is discrete and each nonelementary subgroup generated by two elements of $\phi(G)_{f}$ is discrete, where $f \in \mathcal{H}(\phi(G))$.

Theorem 1.3. Let $G \subset U(1, n ; \mathbb{C})$ be nonelementary. If $\mathcal{P}(\phi(G)) \neq \emptyset$, then $G$ is discrete if and only if $\operatorname{ker}(\phi)$ is discrete and every nonelementary subgroup generated by two elements of $\phi(G)_{f}$ is discrete, where $f \in \mathcal{P}(\phi(G))$.

THEOREM 1.4. Let $G \subset U(1, n ; \mathbb{C})$ be nonelementary and satisfy Condition $C$. If $G$ contains an elliptic element of order at least 3 , then $G$ is discrete if and only if $\operatorname{ker}(\phi)$ is discrete and every nonelementary subgroup generated by two elliptic elements of $G$ is discrete.

Here we say that a subgroup $G$ of $\mathrm{U}(1, n ; \mathbb{C})$ satisfies Condition $C$ if $G$ contains no sequence $\left\{f_{i}\right\}$ such that each $f_{i}$ is parabolic and $f_{i} \rightarrow I_{n+1}$ as $i \rightarrow \infty$.

REMARK 1.5. Theorem 1.1 is a generalization of Theorem 2.1 in [18] to the setting of $\mathrm{U}(1, n, \mathbb{C})$. Theorems 1.2 and 1.3 are the generalizations of [8, Theorem 3.2] and [5, Theorem 1.1]. Theorems 1.2-1.4 are the counterparts in the complex unitary group category of the main results in [19] in $M(n)$.

As applications, by following the line of arguments in [18], we are able to prove the following two theorems concerning the classification of nonelementary subgroups of $\mathrm{U}(1, n, \mathbb{C})$ and the generated system on dense subgroups of $\mathrm{SU}(1, n ; \mathbb{C})$.

THEOREM 1.6. Let $G$ be a nonelementary subgroup of $\mathrm{U}(1, n, \mathbb{C})$. Then either:

(1) $G$ is discrete; or

(2) $\operatorname{ker}(\phi)$ is not discrete but $\phi(G)$ is discrete; or

(3) $\phi(G)$ is dense in $\mathrm{SU}(1, M(G))$.

Here $\mathrm{SU}(1, M(G))$ consists of matrices in $\mathrm{U}(1, M(G))$ with determinant 1.

THEOREM 1.7. Any dense subgroup of $\mathrm{SU}(1, n ; \mathbb{C})$ contains a dense subgroup which is generated by at most $n$ elements if $n \geq 2$ and, if $n=1$, by two elements.

Furthermore, we shall show the following theorem.

THEOREM 1.8. For a nonelementary discrete subgroup $G$ of $\mathrm{SU}(1, n, \mathbb{C}), \mathcal{N}(G)$ is discrete if and only if $M(G)=H_{\mathbb{C}}^{n}$. 
Here $\mathcal{N}(G)$ is the normalizer of $G \subset \mathrm{SU}(1, n ; \mathbb{C})$ in $\mathrm{SU}(1, n ; \mathbb{C})$, that is,

$$
\mathcal{N}(G)=\left\{g \in \mathrm{SU}(1, n ; \mathbb{C}) \mid g G g^{-1}=G\right\}
$$

REMARK 1.9. Theorems 1.6 and 1.7 are the generalizations of [18, Theorems 3.1 and 4.2] to the setting of $\mathrm{U}(1, n, \mathbb{C})$. Theorem 1.8 is a generalization of [20, Theorem 1.1] to the setting of $\operatorname{SU}(1, n, \mathbb{C})$.

This paper is arranged as follows. Section 2 contains some lemmas which are used in Section 3 in the proof of Theorems 1.1-1.4. Section 4 contains the proof of Theorems 1.6 and 1.7. Section 5 contains the proof of Theorem 1.8 and a simple example showing that $G$ can be discrete but $\mathcal{N}(G)$ is not.

\section{Several lemmas}

In order to prove the theorems concerning discreteness of subgroups in $\mathrm{U}(1, n ; \mathbb{C})$, we need the following lemmas.

By the definition of $\phi$, we have the following lemma.

LEMMA 2.1. Let $\phi$ be stated as in (2). Then $G$ is discrete if and only if $\operatorname{ker}(\phi)$ is finite and $\phi(G)$ is discrete.

The following lemma is crucial; see [8].

LEMMA 2.2. Suppose that $f$ and $g \in U(1, n ; \mathbb{C})$ generate a discrete and nonelementary group. Then:

(1) if $f$ is parabolic or loxodromic,

$$
\max \{N(f), N([f, g])\} \geq 2-\sqrt{3},
$$

where $[f, g]=f g f^{-1} g^{-1}$ is the commutator of $f$ and $g, N(f)=\left\|f-I_{n+1}\right\|$;

(2) if $f$ is elliptic,

$$
\max \left\{N(f), N\left(\left[f, g^{i}\right]\right) \mid i=1,2, \ldots, n+1\right\} \geq 2-\sqrt{3} .
$$

LEMMA 2.3 (see [4]). If $g$ is a loxodromic element in $\mathrm{U}(1, n ; \mathbb{C})$ and $f \in U(1, n ; \mathbb{C})$ does not interchange the two fixed points of $g$, then for all large enough $j$, the elements $g^{j} f$ or $g^{-j} f$ are loxodromic.

The following lemma is a classification of elementary subgroups of $\mathrm{U}(1, n ; \mathbb{C})$.

LEMMA 2.4. (1) If $G$ contains a parabolic element but no loxodromic element, then $G$ is elementary if and only if it fixes a point in $\partial H_{\mathbb{C}}^{n}$.

(2) If $G$ contains a loxodromic element, then $G$ is elementary if and only if it fixes a point in $\partial H_{\mathbb{C}}^{n}$ or a point-pair $\{x, y\} \subset \partial H_{\mathbb{C}}^{n}$.

(3) If $G$ is purely elliptic, that is, each nontrivial element of $G$ is elliptic, then $G$ is elementary and fixes a point in $\overline{H_{\mathbb{C}}^{n}}$. 
PROOF. The proof of part (1) is obvious.

For part (2), let $h \in G$ be loxodromic with fixed points $x$ and $y$. It suffices to show that each element of $G$ fixes $\{x, y\}$ setwise if $G$ does not fix a point in $\partial H_{\mathbb{C}}^{n}$. This is obvious by noting that the fixed point set of loxodromic element $f h f^{-1}$ is $\{f(x), f(y)\}$ for $f \in G$ and the definition of elementariness.

For part (3), we claim that the limit set $L(G)$ contains at most one point if $G$ is purely elliptic.

We suppose for the purpose of contradiction that $L(G)=\{x, y\}$. Then there exists a subgroup $G_{1}$ of $G$ with index 2 fixing $\{x, y\}$ pointwise. Therefore each element of $G_{1}$ fixes every point on the geodesic connecting $x$ and $y$, which implies that $L\left(G_{1}\right)=L(G)=\emptyset$. This is a contradiction. If $L(G)$ contains more than two points, then we can find a sequence $g_{m}$ of distinct elements of $G$ such that $g_{m}(z)$ converges uniformly to $x$ on compact subsets of $\overline{H_{\mathbb{C}}^{n}} \backslash\{y\}$, where $x$ and $y$ are distinct points in $L(G)$. Thus for large $m, g_{m}$ are loxodromic, which is a contradiction.

The cases $L(G)=\emptyset$ and $L(G)=\{x\}$ imply that $G$ has a fixed point in $H_{\mathbb{C}}^{n}$ and $\partial H_{\mathbb{C}}^{n}$, respectively, by [7, Lemma 4.3.5] and the $G$-invariance of the limit set. The proof is complete.

LEMMA 2.5. Let $G \subset U(1, n ; \mathbb{C})$ be nonelementary. If each nonelementary subgroup of $G$ generated by two elements of $G_{f}$ is discrete, where $f \in \mathcal{H}(G)$, then $G$ contains no sequence $\left\{f_{i}\right\}$ such that each $f_{i}$ is not elliptic and

$$
f_{i} \rightarrow I_{n+1} \quad \text { as } i \rightarrow \infty \text {. }
$$

PROOF. Suppose for the purposes of contradiction that $G$ does contain such a sequence. Then for large $i$, we may assume that $f_{i}\left(x_{0}\right) \neq y_{0}$ and $f_{i}\left(y_{0}\right) \neq x_{0}$, where $\operatorname{fix}(f)=\left\{x_{0}, y_{0}\right\}$.

Let $g_{i}=f_{i} f f_{i}^{-1}$. Then $g_{i} f^{-1} \rightarrow I_{n+1}$. We infer that, for large enough $i$, the two-generator subgroup $\left\langle f, g_{i}\right\rangle=\left\langle f, g_{i} f^{-1}\right\rangle$ is elementary by Lemma 2.2 and the inequality

$$
N\left(g_{i} f^{-1}\right)+\sum_{k=1}^{n+1} N\left(\left[g_{i} f^{-1}, f^{k}\right]\right)<2-\sqrt{3} .
$$

It follows from $\left\langle f, g_{i} f^{-1}\right\rangle=\left\langle f, g_{i}\right\rangle$ being elementary that fix $(f)=\operatorname{fix}\left(g_{i}\right)$, which implies that

$$
\operatorname{fix}(f) \subset \operatorname{fix}\left(f_{i}\right) .
$$

Let $h \in G$ be loxodromic such that

$$
\operatorname{fix}(f) \cap h[\mathrm{fix}(f)]=\emptyset .
$$

By replacing $f$ by $h f h^{-1}$ in the above discussions, we know that there exists a positive number $M_{1}$ such that for all $i \geq M_{1}$, 


$$
h[\operatorname{fix}(f)] \subset \operatorname{fix}\left(f_{i}\right) .
$$

This shows that $f_{i}$ has at least four fixed points. This is the desired contradiction.

LEMMA 2.6. Let $G \subset U(1, n ; \mathbb{C})$ be nonelementary and contain a parabolic element. If each nonelementary subgroup of $G$ generated by two elements of $G_{f}$ is discrete, where $f \in \mathcal{P}(G)$, then $G$ contains no sequence $\left\{f_{i}\right\}$ such that each $f_{i}$ is not elliptic and

$$
f_{i} \rightarrow I_{n+1} \text { as } i \rightarrow \infty \text {. }
$$

PROOF. Suppose for the purposes of contradiction that $G$ does contain such a sequence $\left\{f_{i}\right\}$. Let $g_{i}=f_{i} f f_{i}^{-1}$ where $f \in \mathcal{P}(G)$. As in the proof of Lemma 2.5, $\left\langle f, g_{i}\right\rangle$ is elementary, which implies that $\operatorname{fix}(f)=\operatorname{fix}\left(g_{i}\right)$, and thus

$$
\operatorname{fix}(f) \subset \operatorname{fix}\left(f_{i}\right) .
$$

Since $G$ is nonelementary, $h_{j} \in G(j=1,2)$ is loxodromic such that

$$
\operatorname{fix}(f) \cap h_{j}[\operatorname{fix}(f)]=\emptyset \quad \text { and } \quad h_{1}[\operatorname{fix}(f)] \cap h_{2}[\operatorname{fix}(f)]=\emptyset .
$$

By replacing $f$ by $h_{j} f h_{j}^{-1}$ in the above discussions, we know that there exists a positive number $M_{1}$ such that for $j=1,2$ and all $i \geq M_{1}$,

$$
h_{j}[\operatorname{fix}(f)] \subset \operatorname{fix}\left(f_{i}\right) .
$$

This shows that $f_{i}$ has at least three fixed points. This is the desired contradiction.

LEMMA 2.7. Let $G$ be nonelementary. If each nonelementary subgroup of $G$ generated by two elliptic elements is discrete and $\operatorname{ker}(\phi)$ is discrete, then $G$ contains no sequence $\left\{f_{i}\right\}$ such that each $f_{i}$ is elliptic and

$$
f_{i} \rightarrow I_{n+1} \quad \text { as } i \rightarrow \infty
$$

PROOF. Suppose for the purposes of contradiction that $G$ contains a sequence $\left\{f_{i}\right\}$ with each $f_{i}$ being elliptic and

$$
f_{i} \rightarrow I_{n+1} \quad \text { as } i \rightarrow \infty
$$

Without loss of generality, we may assume that the smallest totally geodesic submanifold $M(G)$ is $H_{\mathbb{C}}^{k}$, which is a $k$-dimensional complex ball with $\mathbf{S}^{2 k-1}$ as its ideal boundary. Then the set of fixed points of all loxodromic elements lies on $\mathbf{S}^{2 k-1}$. Choose $x_{j} \in L(G)$ and accordingly open balls $\mathrm{U}_{j}$ in $\overline{H_{\mathbb{C}}^{k}}(j=1,2, \ldots, 2 k+1)$ such that $x_{j} \in U_{j}, \mathrm{U}_{j} \cap U_{s}=\emptyset$ whenever $j \neq s$ and, for any $a_{j} \in U_{j}$, there exists only one $(2 k-1)$-dimensional sphere $S\left(a_{1}, \ldots, a_{2 k+1}\right)$ containing $a_{1}, a_{2}, \ldots, a_{2 k+1}$; see [6]. 
Let $h_{j}(j=1,2, \ldots, 2 k+1)$ be loxodromic elements of $G$ such that

$$
\operatorname{fix}\left(h_{1}\right) \subset U_{1},
$$

and the attractive fixed point of $h_{j}$ lies in $\mathrm{U}_{j}$ and the other one lies in $\mathrm{U}_{1}$, where $j=2,3, \ldots, 2 k+1$.

Since $W(G)$ is discrete, after relabelling $\mathrm{U}_{j}, j=1,2, \ldots, 2 k+1$, if necessary, we can choose an elliptic element $f \in G$ of order at least 3 such that

$$
U_{1} \cap \operatorname{fix}\left(f^{2}\right)=\emptyset .
$$

Then there is an integer $t$ such that

$$
\operatorname{fix}\left(h_{j}^{t} f^{2} h_{j}^{-t}\right)=h_{j}^{t}\left(\operatorname{fix}\left(f^{2}\right)\right) \subset U_{j} \quad(j=1,2, \ldots, 2 k+1) .
$$

For large enough $i$ and each $j$,

$$
N\left(f_{i}\right)+\sum_{k=1}^{n+1} N\left(\left[f_{i},\left(h_{t, j}\right)^{k}\right]\right)<2-\sqrt{3},
$$

where $h_{t, j}=h_{j}^{t} f h_{j}^{-t}$.

Combining Lemma 2.2, the above inequality and our assumption yields that

$$
\left\langle f_{i}, h_{t, j}\right\rangle
$$

is elementary.

By Lemma $2.4,\left\langle f_{i}, h_{t, j}\right\rangle$ either fixes a point of $\overline{H_{\mathbb{C}}^{n}}$ or a point-pair $\{x, y\}$ of $\partial H_{\mathbb{C}}^{n}$ setwise. In both cases, the subgroup $\left\langle f_{i}^{2}, h_{t, j}^{2}\right\rangle$ of $\left\langle f_{i}, h_{t, j}\right\rangle$ has a fixed point in $\overline{H_{\mathbb{C}}^{n}}$ by virtue of the fact that $\operatorname{fix}\left(h_{t, j}\right) \subset \operatorname{fix}\left(h_{t, j}^{2}\right)=\operatorname{fix}\left(h_{j}^{t} f^{2} h_{j}^{-t}\right) \subset U_{j}$. Thus it is always the case that $\operatorname{fix}\left(f_{i}^{2}\right) \cap U_{j} \neq \emptyset$. By our choice of $\mathrm{U}_{j}, f_{i}^{2} \in \operatorname{ker}(\phi)$. This is the desired contradiction.

\section{Proof of theorems concerning discreteness}

Proof OF THEOREM 1.1. If $\phi(G)$ is discrete, then each two-generator, nonelementary subgroup $G_{\beta}$ of $\phi(G)$ is discrete. By Lemma 2.1, $\phi\left(G_{\beta}\right)$ is discrete.

For the converse, suppose the contrary. Let $k=\operatorname{dim}(M(G))$. Then there is a sequence of distinct elements $f_{i}$ of $\phi(G) \backslash I_{k+1}$ such that

$$
f_{i} \rightarrow I_{k+1} \quad \text { as } i \rightarrow \infty .
$$

Pass to a subsequence so that fix $\left(f_{i}\right)$ have the Hausdorff limit $X$. Then $X$ is a proper subset of $\overline{M(G)}$. By our choice of sequence, there is a point of $L(G)=L(\phi(G))$ outside $X$. Hence, there is a loxodromic element $h \in \phi(G)$ such that fix $(h) \cap X=\emptyset$. 
We may assume that for large enough $i, f_{i}$ does not interchange the fixed points of $h$ since $f_{i} \rightarrow I_{k+1}$ as $i \rightarrow \infty$. By Lemma 2.3, $h^{j} f_{i}$ is loxodromic for large enough $j$. Thus $h$ and $h^{j} f_{i}$ are two loxodromic elements with distinct fixed points and the twogenerator group $H_{i}=\left\langle h, f_{i}\right\rangle$ is nonelementary in $\phi(G)$. By the definition of the map $\phi, \phi_{H_{i}}(h)$ and $\phi_{H_{i}}\left(h^{j} f_{i}\right)$ are two loxodromic elements in $\phi\left(H_{i}\right) \subset U\left(1, M\left(H_{i}\right)\right)$ with distinct fixed points. Hence, $\phi\left(H_{i}\right)$ is nonelementary and discrete by our assumption. Let $m=\operatorname{dim}\left(M\left(\phi\left(H_{i}\right)\right)\right)$. Applying Lemma 2.2 to $\phi\left(H_{i}\right)=\left\langle\phi_{H_{i}}(h), \phi_{H_{i}}\left(h^{j} f_{i}\right)\right\rangle=$ $\left\langle\phi_{H_{i}}(h), \phi_{H_{i}}\left(f_{i}\right)\right\rangle$, we obtain

$$
\max \left\{N\left(\phi_{H_{i}}\left(f_{i}\right)\right), N\left(\left[\phi_{H_{i}}\left(f_{i}\right), \phi_{H_{i}}(h)^{l}\right]\right) \mid l=1,2, \ldots, m+1\right\} \geq 2-\sqrt{3} .
$$

The above inequality contradicts the fact that $\phi_{H_{i}}\left(f_{i}\right) \rightarrow I_{m+1}$ as $i \rightarrow \infty$. This concludes the proof.

Proof of TheOREM 1.2. The necessity is obvious. For the converse, suppose for the contrary that $G$ is not discrete. Then, by Lemma $2.5, G$ contains a sequence $\left\{f_{i}\right\}$ such that each $f_{i}$ is elliptic and

$$
f_{i} \rightarrow I_{n+1} \quad \text { as } i \rightarrow \infty \text {. }
$$

We shall show that for large enough $i$, each $f_{i}$ belongs to $\operatorname{ker}(\phi)$. This is equivalent to showing that $\phi_{G}\left(f_{i}\right)$ is identity in $\overline{M(G)}$ for large enough $i$.

Let $x \in L(\phi(G))$ and $\mathrm{U}_{x}$ be a small neighbourhood of $x$ in $\overline{M(G)}$. Then there is a loxodromic element $h \in \phi(G)$ such that

$$
\text { fix }(h) \subset U_{x} \text { and } \quad h^{t}[\mathrm{fix}(f)] \subset U_{x}
$$

for all large $t>0$, where $f \in \mathcal{H}(\phi(G))$. For such a loxodromic element $h$, let $g_{t}=h^{t} f h^{-t}$. Then, for large enough $i$,

$$
N\left(\phi\left(f_{i}\right) g_{t} \phi\left(f_{i}\right)^{-1} g_{t}^{-1}\right)+\sum_{k=1}^{n+1} N\left(\left[\phi\left(f_{i}\right) g_{t} \phi\left(f_{i}\right)^{-1} g_{t}^{-1}, g_{t}^{k}\right]\right)<2-\sqrt{3} .
$$

It follows from Lemma 2.2 and the above inequality that

$$
\left\langle g_{t}, \phi\left(f_{i}\right) g_{t} \phi\left(f_{i}\right)^{-1} g_{t}^{-1}\right\rangle=\left\langle h^{t} f h^{-t}, \phi\left(f_{i}\right)\left(h^{t} f h^{-t}\right) \phi\left(f_{i}\right)^{-1}\right\rangle
$$

is elementary. This yields

$$
\operatorname{fix}\left(h^{t} f h^{-t}\right) \subset \operatorname{fix}\left(\phi\left(f_{i}\right)\right)
$$

for large enough $i$, that is,

$$
h^{t}[\operatorname{fix}(f)] \subset \operatorname{fix}\left(\phi\left(f_{i}\right)\right) .
$$

Hence, for any $x \in L(G)$, there exists $M_{x}>0$ such that for all $i>M_{x}$,

$$
\operatorname{fix}\left(\phi\left(f_{i}\right)\right) \cap U_{x} \neq \varnothing .
$$


As in the proof of Lemma 2.7, we choose $2 k+1$ distinct loxodromic fixed points $x_{j}$ such that $x_{j} \in U_{j}, \mathrm{U}_{j} \cap U_{s}=\emptyset$ whenever $j \neq s$ and, for any $a_{j} \in U_{j}$, there exists only one $(2 k-1)$-sphere $S\left(a_{1}, \ldots, a_{2 k+1}\right)$ containing $a_{1}, a_{2}, \ldots, a_{2 k+1}$.

Applying to each $x_{j}$ the above process, we obtain fix $\left(\phi\left(f_{i}\right)\right) \cap U_{x_{j}} \neq \emptyset$ for $i$ large enough. Since the set of fixed points of an elliptic transformation is either a single point or a totally geodesic submanifold by [7, Proposition 3.2.2], there exists $M>0$ such that for all $i \geq M$, the restriction of $f_{i}$ in $\overline{M(G)}$ is identity, that is, $f_{i} \in \operatorname{ker}(\phi)$. This contradicts our assumption that $\operatorname{ker}(\phi)$ is discrete.

Proof of Theorem 1.3. The proof of Theorem 1.3 follows from Lemma 2.6 and the similar arguments to those in the proof of Theorem 1.2.

Proof OF THEOREM 1.4. Suppose that each nonelementary subgroup of $G$ generated by two elliptic elements is discrete and $\operatorname{ker}(\phi)$ is finite, but $G$ is not discrete. Then $G$ contains a sequence $\left\{f_{i}\right\}$ such that

$$
f_{i} \rightarrow I_{n+1} \quad \text { as } i \rightarrow \infty .
$$

We may assume that all $f_{i}$ are not elliptic by Lemma 2.7. Pass to a subsequence if necessary so that fix $\left(f_{i}\right)$ tends in the Hausdorff metric toward a subset of $\partial H_{\mathbb{C}}^{n}$ containing at most two points. After conjugating the sequence $f_{i}$ by a loxodromic element of $G$ if necessary, we can find an elliptic element $g \in G$ of order at least 3 such that

$$
\operatorname{fix}\left(g^{2}\right) \cap \operatorname{fix}\left(f_{i}\right)=\emptyset
$$

for each $i$.

Let $g_{i}=f_{i} g f_{i}^{-1}$. Then $g_{i} g^{-1} \rightarrow I_{n+1}$. We infer that, for large enough $i$, the twogenerator subgroup $\left\langle g, f_{i} g f_{i}^{-1}\right\rangle=\left\langle g, g_{i}\right\rangle=\left\langle g, g_{i} g^{-1}\right\rangle$ is elementary by Lemma 2.2 and the inequality

$$
N\left(g_{i} g^{-1}\right)+\sum_{k=1}^{n+1} N\left(\left[g_{i} g^{-1}, g^{k}\right]\right)<2-\sqrt{3} .
$$

By Lemma 2.4, $\left\langle g, f_{i} g f_{i}^{-1}\right\rangle$ fixes either a point of $\overline{H_{\mathbb{C}}^{n}}$ or a point-pair $\{x, y\}$ of $\partial H_{\mathbb{C}}^{n}$.

If $\left\langle g, f_{i} g f_{i}^{-1}\right\rangle$ has a unique fixed point of $\partial H_{\mathbb{C}}^{n}$ then $\left\{\left[g, f_{i} g f_{i}^{-1}\right]\right\}$ is a sequence of $G$ converging to identity with each $\left[g, f_{i} g f_{i}^{-1}\right]$ being either elliptic or parabolic by [10, Proposition 1.11]. By our assumption, this is a sequence of elliptic elements of $G$ converging to identity. This is a contradiction by Lemma 2.7.

Suppose that $\left\langle g, f_{i} g f_{i}^{-1}\right\rangle$ fixes either a point of $H_{\mathbb{C}}^{n}$ or a point-pair $\{x, y\}$ of $\partial H_{\mathbb{C}}^{n}$. Note that $\operatorname{fix}(g) \subset \operatorname{fix}\left(g^{2}\right)$. For both cases, the subgroup $\left\langle g^{2}, f_{i} g^{2} f_{i}\right\rangle$ of $\left\langle g, f_{i} g f_{i}^{-1}\right\rangle$ has a fixed point in $H_{\mathbb{C}}^{n}$. Thus $f_{i} g^{2} f_{i}^{-1} g^{-2}$ is a sequence of elliptic elements of $G$ converging to identity by noting that $f_{i} g^{2} f_{i}^{-1} g^{-2} \neq I_{n+1}$ for all $i$. This is a contradiction by Lemma 2.7. The proof is complete. 


\section{Applications of discreteness criteria}

Proof of TheOREM 1.6. By Lemma 2.1, it suffices to prove that the nondiscrete subgroup $\phi(G) \in U(1, M(G))$ is dense in $\operatorname{SU}(1, M(G))$. By the definition of $\phi$ and the fact that $G$ is nonelementary, we know that there is no point of $\overline{M(G)}$ and no proper totally geodesic submanifold in $M(G)$ which is invariant under $\phi(G)$. It follows from [7, Theorem 4.5.1 or Theorem 4.4.2] that $\phi(G)$ is dense in $\operatorname{SU}(1, M(G))$.

By Theorems 1.1 and 1.6, the following corollary holds.

Corollary 4.1. Let $G$ be a dense subgroup of $\mathrm{U}(1, n ; \mathbb{C})$. Then $G$ contains a twogenerator nonelementary subgroup $F$ such that $\phi_{F}(F)$ is dense in $\mathrm{SU}(1, M(F))$.

Proof of Theorem 1.7. Let $G$ be a dense subgroup of $\operatorname{SU}(1, n ; \mathbb{C})$. If $n=1$, the result is obvious by [18] and the fact $\operatorname{SU}(1,1 ; \mathbb{C})$ being a subgroup of $\operatorname{SL}(2, \mathbb{C})$ (see $[3,7]$ for details). By this corresponding, we know the correctness in the case $n=1$.

We assume that $n \geq 2$ in what follows. By Corollary 4.1, we know that there is a two-generator subgroup $F$ such that $\phi_{F}(F)$ is a dense subgroup in $\mathrm{SU}(1, M(F))$.

Since $F$ is nonelementary, the cardinality of the limit set $L(F)$ of $f$ is greater than 3 and thus $\operatorname{dim}(M(F))>1$. We mention that, in contrast to the real hyperbolic space, the convex hull of three (generic) points in the complex hyperbolic space may have dimension 4.

We pick a loxodromic $g \in G$ whose fixed points are outside $\overline{M(F)}$. Let $F_{1}$ be threegenerator subgroup generated by $F$ and $g$. Then $M(F)$ is a proper subset of $M\left(F_{1}\right)$ and $\phi\left(F_{1}\right)$ is dense in $\mathrm{SU}\left(1, M\left(F_{1}\right)\right)$ by Theorem 1.6. We know that $\operatorname{dim}\left(M\left(F_{1}\right)\right)$ is at least 3 .

By repeating the above reasoning a finite number of times, we get a subgroup $H$ of $G$ which is generated by at most $n$ elements and is dense in $\operatorname{SU}(1, n ; \mathbb{C})$. This completes the proof.

\section{Discreteness of the normalizer of $G$}

We first mention that each $e^{i \theta} I_{n+1}(\theta \in \mathbb{R})$ belongs to the normalizer of $G \in$ $U(1, n ; \mathbb{C})$ in $\mathrm{U}(1, n ; \mathbb{C})$, which implies the nondiscreteness of the normalizer of $G$ in $\mathrm{U}(1, n ; \mathbb{C})$. Therefore we restrict our discussions to $\mathrm{SU}(1, n ; \mathbb{C})$. Before proving Theorem 1.8, we give a simple example to show that $G$ can be discrete but $\mathcal{N}(G)$ is not.

EXAMPLE 5.1. Let $G_{1}$ be any discrete nonelementary subgroup of $\mathrm{SU}(1, n ; \mathbb{C})$ and

$$
G=\left\{h=\left(\begin{array}{cc}
g & \\
& 1
\end{array}\right) \mid g \in G_{1}\right\} \subset \mathrm{SU}(1, n+1 ; \mathbb{C}) .
$$


Then $G$ is a discrete and nonelementary subgroup of $\mathrm{SU}(1, n+1 ; \mathbb{C})$ and the set

$$
\begin{aligned}
& D(G)=\left\{h=\left(\begin{array}{ll}
e^{-i \theta /(n+1)} f & \\
& e^{i \theta}
\end{array}\right) \mid f \in N\left(G_{1}\right), \forall \theta \in \mathbb{R}\right\} \\
& \subset \mathcal{N}(G) \subset \mathrm{SU}(1, n+1 ; \mathbb{C}) \text {. }
\end{aligned}
$$

It is clear that $D(G)$ is not discrete.

In particular, for a purely loxodromic subgroup $G_{1}$ of $\operatorname{SU}(1, n ; \mathbb{C}), D(G)=\mathcal{N}(G)$ in the above example. In fact let

$$
f=\left(\begin{array}{ll}
A & \alpha \\
\beta & d
\end{array}\right) \in \mathcal{N}(G),
$$

where $A$ is an $(n+1) \times(n+1)$ complex matrix, $\alpha$ is an $(n+1) \times 1$ complex matrix and $\beta$ is a $1 \times(n+1)$ complex matrix. Then there exist two elements $g_{1}, g_{2} \in G_{1}$ such that

$$
f h_{1}=h_{2} f
$$

where $h_{1}=\operatorname{diag}\left(g_{1}, 1\right)$ and $h_{2}=\operatorname{diag}\left(g_{2}, 1\right)$ are two elements of $G$. Thus we have that

$$
A g_{1}=g_{2} A, \quad g_{2} \alpha=\alpha, \quad \beta g_{1}=\beta .
$$

Since each nontrivial element of $G_{1}$ does not have 1 as its eigenvalue, $\alpha=0$ and $\beta=0$, which implies that $D(G)=\mathcal{N}(G)$ in this case.

We mention that $f=\operatorname{diag}\left(e^{-i \theta /(n+1)} I_{n+1}, e^{i \theta}\right) \in \operatorname{ker}\left(\phi_{\mathcal{N}(G)}\right)$, which implies that $\operatorname{ker}\left(\phi_{\mathcal{N}(G)}\right)$ is not discrete in the above case.

The above observation motivates the following proposition.

Proposition 5.2. For a nonelementary subgroup $G$ of $\mathrm{SU}(1, n, \mathbb{C}), \mathcal{N}(G)$ is discrete if and only if both $G$ and $\operatorname{ker}\left(\phi_{\mathcal{N}(G)}\right)$ are discrete.

Proof. The necessity is obvious. For the converse, suppose that both $G$ and $\operatorname{ker}\left(\phi_{\mathcal{N}(G)}\right)$ are discrete but $\mathcal{N}(G)$ is not discrete. Then there exists a distinct sequence $\left\{f_{j}\right\}$ of $\mathcal{N}(G)$ such that

$$
f_{i} \rightarrow I_{n+1} \quad \text { as } i \rightarrow \infty .
$$

Note the discreteness of $G$ and the commutator $\left[f_{i}, g\right]:=f_{i} g f_{i}^{-1} g^{-1} \in G$ for any $g \in G$,

$$
\left[f_{i}, g\right]=I_{n+1}
$$

for large enough $i$. Hence $\operatorname{fix}(g)=f_{i}(\operatorname{fix}(g))$, which implies that if $g$ is loxodromic then

$$
\operatorname{fix}(g) \subset \operatorname{fix}\left(f_{i}\right)
$$

for large enough $i$. 
Since $G$ is a normal subgroup of $\mathcal{N}(G)$ and $\mathcal{N}(G)$ does not have a common fixed point in $\partial H_{\mathbb{C}}^{n}, L(G)=L(\mathcal{N}(G))$ by [7, Lemma 4.3.4]. This implies that $f_{i} \in \operatorname{ker}\left(\phi_{\mathcal{N}(G)}\right)$ for large enough $i$. This contradicts the fact that $\operatorname{ker}\left(\phi_{\mathcal{N}(G)}\right)$ is discrete. The proof is complete.

PROOF OF THEOREM 1.8. If $M(G) \neq H_{\mathbb{C}}^{n}$, we may assume without loss of generality that $M(G)=H_{\mathbb{C}}^{k}$ with $2 \leq k \leq n-1$. Then $G$ is of the form

$$
G=\left\{g=\left(\begin{array}{ll}
g_{1} & \\
& A
\end{array}\right)\right\} \subset \mathrm{SU}(1, n ; \mathbb{C}),
$$

where $g_{1} \in U(1, k+1 ; \mathbb{C}), A \in U(n-k)$, the set of all $(n-k) \times(n-k)$ unitary matrices. Note that for each $\theta \in \mathbb{R}$,

$$
f=\left(\begin{array}{ll}
\exp (-i(n-k) \theta /(k+1)) I_{k+1} & \\
& e^{i \theta} I_{n-k}
\end{array}\right) \in \operatorname{ker}\left(\phi_{\mathcal{N}(G)}\right) .
$$

This implies that $\operatorname{ker}\left(\phi_{\mathcal{N}(G)}\right)$ is not discrete. Thus $\mathcal{N}(G)$ is not discrete by Proposition 5.2.

If $M(G)=H_{\mathbb{C}}^{n}$ then $\operatorname{ker}\left(\phi_{G}\right)=\operatorname{ker}\left(\phi_{\mathcal{N}(G)}\right)=\left\{I_{n+1}\right\}$, which implies that $\mathcal{N}(G)$ is discrete by Proposition 5.2. The proof is complete.

\section{References}

[1] B. N. Apanasov, 'Geometry and topology of complex hyperbolic and Cauchy-Riemannian manifolds', Russian Math. Surveys 52(5) (1997), 895-928.

[2] A. Basmajian and R. Miner, 'Discrete subgroups of complex hyperbolic motions', Invent. Math. 131(1) (1998), 85-136.

[3] W. Cao, J. R. Parker and X. Wang, 'On the classification of quaternion Möbius transformations', Math. Proc. Cambridge Philos. Soc. 137(2) (2004), 349-361.

[4] W. Cao and X. Wang, 'Geometric characterizations for subgroups of $P U(1, n ; \mathbb{C})$ ', Northeast. Math. J. 21(1) (2005), 45-53.

[5] - 'Discreteness criteria and algebraic convergence theorem for subgroups in $P U(1, n ; \mathbb{C})$ ', Proc. Japan Acad. 82(3) (2006), 49-52.

[6] M. Chen, 'Discreteness and convergence of Möbius groups', Geom. Dedicata 104 (2004), 61-69.

[7] S. S. Chen and L. Greenberg, 'Hyperbolic spaces', in: Contributions to Analysis: A Collection of Papers Dedicated to Lipman Bers (eds. L. V. Ahlfors et al.) (Academic Press, New York, 1974), pp. 49-87.

[8] B. Dai, A. Fang and B. Nai, 'Discreteness criteria for subgroups in complex hyperbolic space', Proc. Japan Acad. 77(10) (2001), 168-172.

[9] T. Jørgensen, 'On discrete groups of Möbius transformations', Amer. J. Math. 98(3) (1976), 739749.

[10] S. Kamiya, 'Notes on elements of U(1, $n ; \mathbb{C})$ ', Hiroshima Math. J. 21(1) (1991), 23-45.

[11] S. Katok, Fuchsian Groups (University of Chicago Press, Chicago, 1992).

[12] A. Korányi and H. M. Reimann, 'Quasiconformal mappings on the Heisenberg group', Invent. Math. 80(2) (1985), 309-338.

[13] B. Maskit, Kleinian Groups (Springer, Berlin, 1994).

[14] G. J. Martin, 'On discrete Möbius groups in all dimensions', Acta Math. 163(1) (1989), 253-289.

[15] J. R. Parker, 'Uniform discreteness and Heisenberg translations', Math. Z. 225(3) (1997), 485-505. 
[16] J. Ratcliffe, 'On the isometry groups of hyperbolic manifolds', Contemp. Math. 169 (1994), 491495.

[17] D. Sullivan, 'Quasiconformal homeomorphisms and dynamics: Structural stability implies hyperbolicity for Kleinian groups', Acta Math. 155(3) (1985), 243-260.

[18] X. Wang, 'Dense subgroups of $n$-dimensional Möbius groups', Math. Z. 243(4) (2003), 643-651.

[19] X. Wang, L. Li and W. Cao, 'Discreteness criteria for Möbius groups acting on $\bar{R}^{n}$, Israel J. Math. 150 (2005), 357-368.

[20] X. Wang, Y. Li and M. Xia, 'The discreteness of the normalizers of higer dimensional Kleinian groups and the isomorphisms between Kleinian groups induced by quasiconformal mappings', Glasg. Math. J. 47(2) (2005), 373-378.

WENSHENG CAO, Department of Mathematics and Physics, Wuyi University, Jiangmen 529020, China

e-mail: wenscao@yahoo.com.cn 